\title{
Aptidão física de idosos com doença de Parkinson submetidos à intervenção pelo método Pilates
}

\author{
Vanessa Santiago do Carmo*, Lorena Aragão Vilas Boas", \\ Ana Luíza Azevedo do Vale" ", Igor de Matos Pinheiro"**"
}

\section{Resumo}

A doença de Parkinson (DP) é uma doença neurodegenerativa que provoca deterioração dos parâmetros físicos dos idosos, resultando em limitação funcional e redução da aptidão física. O método Pilates é uma modalidade de exercício físico que promove benefícios para os idosos, porém, observa-se uma escassez de publicações científicas sobre o impacto da técnica nos parâmetros da aptidão física em idosos com DP. O objetivo deste estudo foi examinar a aptidão física de idosos com DP submetidos à intervenção pelo método Pilates. Trata-se de um ensaio clínico de braço único, realizado com quatro idosos acompanhados no ambulatório de fisioterapia de um centro de geriatria e gerontologia em Salvador, Bahia, Brasil. Participaram idosos ( $\geq 60$ anos) com diagnóstico de DP, em uso regular das medicações, que não houvessem realizado atividade física nos dois meses anteriores à intervenção.
Foram excluídos idosos com declínio cognitivo, com disfunções musculoesqueléticas que comprometessem a realização dos exercícios ou com frequência irregular na intervenção (três ausências consecutivas). Os dados coletados foram: situação clínica e sociodemográfica, estado cognitivo, severidade da DP e aptidão física. Os idosos participaram de 30 sessões do método Pilates (pré-Pilates, mat Pilates, treino de equilíbrio e marcha, exercícios de relaxamento corporal). Após a intervenção, foram observados valores superiores aos da avaliação inicial nos parâmetros de força e resistência de membros superiores e inferiores, de flexibilidade de membros superiores e inferiores e de mobilidade física. Conclui-se que o método Pilates pode ser uma ferramenta eficaz no processo de reabilitação desses idosos parkinsonianos.

Palavras-chave: Aptidão física. Doença de Parkinson. Idosos. Pilates.

* Fisioterapeuta assistencial do Ambulatório de Fisioterapia do Centro de Geriatria e Gerontologia Júlia Magalhães das Obras Sociais Irmã Dulce. E-mail: fta.vanessa@gmail.com

** Fisioterapeuta assistencial do Ambulatório de Fisioterapia do Centro de Geriatria e Gerontologia Júlia Magalhães das Obras Sociais Irmã Dulce. Especialista em Fisioterapia Cardiovascular e Respiratória. E-mail: lorearagao@gmail.com

*** Fisioterapeuta principal do Centro de Geriatria e Gerontologia Júlia Magalhães das Obras Sociais Irmã Dulce. E-mail: ana.azevedo@irmadulce.org.br

***** Mestre em Processos Interativos dos Órgãos e Sistemas pela Universidade Federal da Bahia, fisioterapeuta da Educação Permanente das Obras Sociais Irmã Dulce do Centro de Geriatria e Gerontologia Júlia Magalhães das Obras Sociais Irmã Dulce. Endereço para correspondência: Av. Bonfim, 161, Largo de Roma, Salvador, Bahia, Brasil. CEP: 40420-415. E-mail: igordematospinheiro@gmail.com

$\rightarrow$ http://dx.doi.org/10.5335/rbceh.v14i2.7006

Recebido em: 04.05.2017. Aceito em: 06.12.2017. 


\section{Introdução}

O envelhecimento é um processo fisiológico de caráter individual, influenciado por fatores genéticos e pelo estilo de vida, que envolve alterações fisiológicas nas estruturas, com repercussão na funcionalidade e na aptidão física do indivíduo (PEREIRA et al., 2009; VILA et al., 2013). A aptidão física é um estado dinâmico de energia e vitalidade que está intimamente relacionado com a capacidade de executar as atividades de vida diária em condições aeróbicas adequadas, com força e flexibilidade muscular, mantendo a autonomia e a saúde dos indivíduos (CIPRIANI et al., 2010; NOGUEIRA et al., 2010; SEEMANN et al., 2016).

A prática regular de exercício físico promove prevenção de doenças crônicas, possibilita manutenção da capacidade funcional, reduz morbidade e mortalidade, além de trazer benefícios nos componentes mental, psicológico e social do indivíduo (NOGUEIRA et al., 2010; REIS; MASCARENHAS; LYRA, 2011; SEEMANN et al., 2016). Os idosos que realizam exercício físico regular apresentam melhores níveis de atenção, independência funcional, redução do risco de quedas, melhor aptidão física, com impactos positivos na qualidade de vida quando comparados a idosos sedentários (PEREIRA et al., 2009; ALENCAR et al., 2010; REIS; MASCARENHAS; LYRA, 2011; VILA et al., 2013).

O método Pilates é uma modalidade de exercício físico que traz benefícios para os idosos, melhorando as capacida- des físicas, como equilíbrio, flexibilidade, força muscular, consciência cinestésica, controle e alinhamento postural (NASCIMENTO; LIMA, 2013; GUIMARÃES et al., 2014). O método é composto de exercícios de baixo impacto que consideram a relação entre o corpo e a disciplina mental, seguindo os princípios de respiração, centralização, concentração, controle, precisão e fluidez do movimento (LIMA et al., 2009; MARÉS et al., 2012; NASCIMENTO; LIMA, 2013; PINHEIRO, K. et al., 2014). A técnica de Pilates pode ser realizada no solo (mat Pilates), com acessórios ou aparelhos específicos, adaptados conforme a necessidade de cada praticante (GONÇALVES; ANGELO; MARTINS, 2009; MARÉS et al., 2012; COSTA et al., 2016). O método pode ser utilizado para o condicionamento físico, para a prevenção e a reabilitação de pacientes com desordens neurológicas, ortopédicas, reumatológicas ou respiratórias (LIMA et al., 2010; DA ROSA et al., 2013; COSTA et al., 2016).

A doença de Parkinson (DP) é a segunda doença neurodegenerativa mais comum no idoso, afetando uma em cada cem pessoas acima de 75 anos de idade. Com o aumento da expectativa de vida, estima-se que por volta de 2020 a doença acometa mais de 40 milhões de pessoas no mundo (FREITAS et al., 2006; LIMA et al., 2010; QUINTELLA et al., 2013; PINHEIRO, I. et al., 2014). A DP acomete o sistema nigroestriataldopaminérgico e clinicamente é caracterizada por distúrbios do movimento, da postura e do equilíbrio, manifestando-se por meio de acinesia, rigidez, tremor de repouso 
e instabilidade postural (FREITAS et al., 2006; SANT et al., 2008; LIMA et al., 2010). Com a progressão da doença, há deterioração dos parâmetros físicos, como força e resistência muscular, equilíbrio, flexibilidade, agilidade e coordenação, o que resulta em limitação funcional e redução da aptidão física. Outras alterações não motoras, como disfunção cognitiva, depressão, alterações gastrointestinais e sintomas autonômicos, podem fazer parte da vida desses indivíduos em graus variados (FREITAS et al., 2006; KING; HORAK, 2009; LIMA et al., 2009; JOHNSON et al., 2013; FREITAS; ZAGER; CAMPBELL, 2015).

As disfunções motoras nos idosos com DP devem ter uma abordagem precoce, a fim de amenizar ou retardar o aparecimento dos sintomas e a progressão da doença, de forma que possibilite manutenção da independência e da autonomia do idoso (LIMA et al., 2010). Existem diversas abordagens descritas na literatura para tratamento das disfunções motoras da DP nos idosos, entre elas, o método Pilates vem apresentando resultados promissores (ROYER; WALDMANN, 2007; KING; HORAK, 2009; LIMA et al., 2010). Segundo Johnson et al. (2013), idosos parkinsonianos submetidos a seis semanas de intervenção com exercícios de Pilates apresentaram melhora na postura, nos padrões de marcha e no equilíbrio. De Freitas, Zager e Campbell (2015) observaram melhora do equilíbrio, redução do risco de quedas e melhora da qualidade de vida após 24 sessões de Pilates realizadas com idosa parkinsoniana.
Atualmente, o método Pilates tem sido aplicado em diversas populações; para melhor compreensão dos seus efeitos, porém, observa-se uma escassez de publicações científicas sobre o impacto do método Pilates nos parâmetros da aptidão física em idosos com DP. Dessa forma, o presente estudo teve como objetivo examinar a aptidão física de idosos com DP submetidos à intervenção pelo método Pilates.

\section{Metodologia}

O presente estudo trata de um ensaio clínico de braço único, realizado com idosos com DP submetidos à intervenção pelo método Pilates. Participaram do estudo idosos acompanhados no ambulatório de fisioterapia de um centro de geriatria e gerontologia em Salvador, Bahia, Brasil. A seleção amostral ocorreu por conveniência, de acordo com a disponibilidade do participante em comparecer ao local de tratamento semanalmente nos dias e horários programados. Este estudo foi aprovado no Comitê de Ética em Pesquisa (Parecer no ${ }^{\circ}$ 1.686.161), e todos os participantes assinaram o termo de consentimento livre e esclarecido.

Foram incluídos os pacientes com idade igual ou superior a 60 anos, com diagnóstico de DP, que apresentassem estadiamento da doença entre 1,5 e 2,5 de acordo com a escala Hoehn and Yahr modificada -, em uso regular das medicações para a DP, e que não tivessem realizado atividade física nos últimos dois meses anteriores à intervenção. Foram 
excluídos os idosos com declínio cognitivo, com disfunções musculoesqueléticas que comprometessem a realização dos exercícios ou com frequência irregular (três ausências consecutivas).

Antes e após a intervenção pelo método Pilates, foram coletados os seguintes dados dos idosos:

a) dados clínicos e sociodemográficos: idade, sexo, escolaridade, estado civil, diagnóstico clínico e medicações em uso;

b) dados cognitivos: estado cognitivo avaliado por meio do Mini-Exame do Estado Mental (Meem). Os pontos de corte foram ajustados de acordo com a escolaridade do idoso: 18 pontos - analfabeto; 21 pontos - 1 a 3 anos de escolaridade; 24 pontos - 4 a 7 anos; 26 pontos mais de 7 anos (BERTOLUCCI et al., 1994; LOURENÇO; VERAS, 2006);

c) dados da severidade da DP: grau da doença avaliado de acordo com a escala Hoehn and Yahr modificada para classificação do nível de incapacidade (estágio 0 - nenhum sinal da doença; estágio 1 - doença unilateral; estágio 1,5 - envolvimento unilateral e axial; estágio 2 - doença bilateral sem déficit de equilíbrio; estágio 2,5 - doença bilateral leve, com recuperação no teste do empurrão; estágio 3 doença bilateral leve a moderada, com instabilidade postural e inca- pacidade para viver independentemente; estágio 4 - incapacidade grave, incapaz de caminhar ou permanecer de pé sem ajuda; estágio 5 - confinamento a cama ou cadeira de rodas) (GOULART et al., 2004; GOULART; PEREIRA, 2005; MELLO; BOTELHO, 2010);

d) dados da aptidão física: bateria de testes de aptidão física Senior Fitness Test. Este instrumento foi desenvolvido para avaliar os principais parâmetros físicos associados à capacidade funcional de idosos independentes de 60 a 90 anos, com valores normativos por grupos etários e sexo, sendo possível a comparação de resultados (RIKLI; JONES, 2002; LOBO; PEREIRA, 2007; VILA et al., 2013).

A intervenção foi realizada em grupo por uma fisioterapeuta com formação no método Pilates. As sessões duraram 60 minutos e ocorreram 3 vezes na semana, durante 10 semanas. Todos os participantes foram orientados com relação aos princípios da técnica e ao uso regular da medicação para a DP, conforme prescrição médica. A intensidade e a progressão dos exercícios foram determinadas de acordo com a aptidão física de cada idoso, e, para melhor aprendizado motor e execução dos movimentos, a intervenção pelo método Pilates foi fragmentada em quatro momentos: 
1) pré-Pilates: alinhamento postural; alongamentos de cadeias anterior, posterior e lateral; movimentos circulares de ombros em ortostase; treino de transferência (ortostase - quatro apoios - decúbito dorsal); treino respiratório diafragmático; dissociação de cinturas; exercícios ativos livres e resistidos em membros; exercício isométrico de adutores com contração simultânea dos músculos abdominais, dos glúteos e da musculatura do períneo, de modo a perceber o powerhouse; posição table top com joelhos e coxofemorais a 90 graus sem tocar os pés no chão;

2) mat Pilates: pelvic curl (exercício para mobilização de coluna, fortalecimento de glúteos, musculatura posterior da perna e abdômen), one leg circle com auxílio da faixa elástica (círculos com a perna estendida, fortalecimento de oblíquo externo e reto abdominal e eretores da espinha), teaser com auxílio da faixa elástica (formar um $\mathrm{V}$ com tronco e membros inferiores, fortalecimento do reto abdominal e oblíquo externo), side kicks (chutes laterais, fortalecimento de abdômen associado a flexão e extensão do quadril), one leg kick (chute com uma perna em decúbito ventral, fortalecimento de isquiotibiais, eretores da espinha e glúteos), swan (alongamento da cadeia anterior do tronco e mobilização da coluna, fortalecimento de peitoral maior, tríceps braquial, ancôneo e deltoide anterior), spine twist com auxílio do bastão (alongamento dos rotadores de tronco, isquiotibiais, quadrado lombar, fortalecimento do reto abdominal, oblíquo externo e interno);

3) treino de equilíbrio e marcha: apoio unipodal com olhos abertos e com olhos fechados, base de suporte diminuída com pés juntos, um pé na frente do outro, giro em seu próprio eixo e marcha estacionária;

4) exercícios de relaxamento corporal, associando treino respiratório diafragmático.

Os dados do estudo foram analisados por meio da análise descritiva simples e qualitativa.

\section{Resultados}

A amostra foi composta inicialmente por 15 idosos; 9 foram excluídos por apresentarem déficit cognitivo e disfunções musculoesqueléticas que comprometiam a realização dos exercícios; e 2 , por frequência irregular. A amostra final, portanto, foi composta por 4 idosos, cujas características sociodemográficas e clínicas são apresentadas na Tabela 1. 
Tabela 1 - Caracterização sociodemográfica e clínica dos idosos

\begin{tabular}{l|l|l|l|l}
\hline \multicolumn{1}{c|}{ Variáveis } & \multicolumn{1}{|c|}{ Paciente 1 } & \multicolumn{1}{c|}{ Paciente 2 } & \multicolumn{1}{c|}{ Paciente 3} & \multicolumn{1}{c}{ Paciente 4 } \\
\hline Idade & 69 anos & 78 anos & 64 anos & 83 anos \\
Sexo & Masculino & Masculino & Masculino & Masculino \\
Estado civil & Casado & Casado & Casado & Viúvo \\
Escolaridade & $2^{\circ}$ grau completo & 1 o grau completo & $2^{\circ}$ grau completo & 1 grau incompleto \\
Meem & 28 & 22 & 29 & 19 \\
$\begin{array}{l}\text { Hoehn and Yahr } \\
\text { modificada }\end{array}$ & 2,5 & 1,5 & 1,5 & 1,5 \\
\hline
\end{tabular}

Fonte: elaboração dos autores.

Nas Tabelas 2, 3, 4 e 5, encontram-se descritos a avaliação da aptidão física dos idosos participantes deste estudo e os valores de antes e depois da interven- ção pelo método Pilates. Os dados foram comparados aos valores de referência estabelecidos por Rikli e Jones (2002), de acordo com a faixa etária do indivíduo.

Tabela 2 - Avaliação da aptidão física do paciente 1 com DP antes e depois da intervenção pelo método Pilates

\begin{tabular}{l|c|c|c}
\hline \multicolumn{1}{c|}{ Paciente 1 } & Pré & Pós & VR - 65 a 69 anos \\
\hline Força MMII (repetições) & 10 & 12 & $12-18$ \\
Força MMSS (repetições) & 13 & 19 & $15-21$ \\
Flexibilidade MI (cm +/-) & -2 & 0 & $-3-+3$ \\
Flexibilidade MS (cm +/-) & -15 & -15 & $-7,5--1$ \\
TME 2' (número de passos) & 59 & 58 & $86-116$ \\
Caminhada 2,44 m (segundos) & 14 & 9 & $5.7-4.3$ \\
\hline
\end{tabular}

Fonte: elaboração dos autores.

Legenda: VR - valores de referência; MMII - membros inferiores; MMSS - membros superiores; MI - membro inferior; MS - membro superior; TME 2' - teste marcha estacionária de 2 minutos.

Tabela 3 - Avaliação da aptidão física do paciente 2 com DP antes e depois da intervenção pelo método Pilates

\begin{tabular}{l|c|c|c}
\hline \multicolumn{1}{c|}{ Paciente 2 } & Pré & Pós & VR - 75 a 79 anos \\
\hline Força MMII (repetições) & 12 & 9 & $11-17$ \\
Força MMSS (repetições) & 17 & 20 & $13-19$ \\
Flexibilidade MI (cm +/-) & 12,5 & 14 & $-4-+2$ \\
Flexibilidade MS (cm +/-) & -47 & -24 & $-9--2$ \\
TME 2' (número de passos) & 80 & 57 & $73-109$ \\
Caminhada 2,44 m (segundos) & 12 & 8 & $7.2-4.6$ \\
\hline
\end{tabular}

Fonte: elaboração dos autores.

Legenda: VR - valores de referência; MMII - membros inferiores; MMSS - membros superiores; MI - membro inferior; MS - membro superior; TME 2' - teste marcha estacionária de 2 minutos. 
Tabela 4 - Avaliação da aptidão física do paciente 3 com DP antes e depois da intervenção pelo método Pilates

\begin{tabular}{l|c|c|c}
\hline \multicolumn{1}{c|}{ Paciente 3 } & Pré & Pós & VR - 60 a 64 anos \\
\hline Força MMII (repetições) & 14 & 13 & $14-19$ \\
Força MMSS (repetições) & 21 & 27 & $16-22$ \\
Flexibilidade MI (cm +/-) & 16 & 18 & $-2,5-+4$ \\
Flexibilidade MS (cm +/-) & 0 & 4 & $-6,5-+0$ \\
TME 2' (número de passos) & 102 & 98 & $87-115$ \\
Caminhada 2,44 m (segundos) & 10 & 6 & $5.6-3.8$ \\
\hline
\end{tabular}

Fonte: elaboração dos autores.

Legenda: VR - valores de referência; MMII - membros inferiores; MMSS - membros superiores; MI - membro inferior; MS - membro superior; TME 2' - teste marcha estacionária de 2 minutos.

Tabela 5 - Avaliação da aptidão física do paciente 4 com DP antes e depois da intervenção pelo método Pilates

\begin{tabular}{l|c|c|c}
\hline \multicolumn{1}{c|}{ Paciente 3 } & Pré & Pós & VR -80 a 84 anos \\
\hline Força MMII (repetições) & 10 & 11 & $10-15$ \\
Força MMSS (repetições) & 12 & 14 & $13-19$ \\
Flexibilidade MI (cm +/-) & -16 & -10 & $-5,5-+1,5$ \\
Flexibilidade MS (cm +/-) & -21 & $-7,5$ & $-9,5--2,0$ \\
TME 2' (número de passos) & 94 & 102 & $71-103$ \\
Caminhada 2,44 m (segundos) & 11 & 9 & $7.6-5.2$ \\
\hline
\end{tabular}

Fonte: elaboração dos autores.

Legenda: VR - valores de referência; MMII - membros inferiores; MMSS - membros superiores; MI - membro inferior; MS - membro superior; TME 2' - teste marcha estacionária de 2 minutos.

Antes da intervenção pelo método Pilates, o paciente 1 não apresentou força de membros inferiores adequada à sua faixa etária, conforme os valores de normalidade estabelecidos por Rikli e Jones (2002); assim como os pacientes 1 e 4 não apresentaram força de membros superiores. Após a intervenção, os idosos 1 e 4 mostraram valores superiores de força muscular de membros inferiores, e todos os idosos apresentaram valores superiores de força muscular de membros superiores.
Em relação à flexibilidade, todos os pacientes demonstraram melhores valores de flexibilidade de membros inferiores e superiores, quando comparados aos dados coletados na pré-intervenção, exceto o paciente 1 , que manteve sua flexibilidade de membro superior.

Os pacientes 2, 3 e 4 apresentaram valores do TME 2' dentro dos valores de referência antes da intervenção. Após a intervenção pelo método Pilates, o paciente 4 otimizou seu desempenho, e o paciente 3 teve uma redução de desempenho, porém, manteve-se dentro 
do valor de normalidade para sua faixa etária. Na realização do teste de caminhada, todos os pacientes mostraram valores inferiores aos de referência antes da intervenção; e, apesar da melhora do desempenho neste teste, nenhum idoso conseguiu atingir o valor de referência após a intervenção.

\section{Discussão}

Os resultados encontrados neste estudo demonstram que os idosos com DP apresentaram melhores parâmetros da aptidão física após a intervenção fisioterapêutica com o método Pilates. A amostra deste estudo foi composta por homens com idades entre 64 e 83 anos, corroborando os estudos de Werneck e Alvarenga (1999) e Quintella et al. (2013), que afirmaram que há uma incidência maior da DP em homens do que em mulheres a partir da sexta década de vida.

No estudo realizado por Galhardo, Amaral e Vieira (2009), observou-se que o baixo nível educacional correlaciona-se positivamente para o declínio cognitivo na DP. No presente estudo, foi constatada uma menor pontuação no Meem no idoso com menor nível de escolaridade e maior idade. Esses resultados são similares aos encontrados no estudo de Lourenço e Veras (2006).

A aptidão física dos idosos com DP deste estudo apresentou-se de forma heterogênea, apesar de a maioria dos participantes demonstrar comprometimentos semelhantes, de acordo com a escala de Hoehn and Yahr modificada. Acredita-se que tal fato seja reflexo do processo de envelhecimento que é específico em cada indivíduo e do estilo de vida adotado por cada paciente, conforme demonstrado por Vila et al. (2013), em estudo com idosas praticantes de exercício físico e idosas sedentárias, observando que as idosas que realizavam exercício físico apresentaram melhor aptidão física $\mathrm{e}$ maior nível de atenção, se comparadas às idosas sedentárias.

Os valores pré e pós-intervenção encontrados sinalizam uma otimização dos parâmetros de força muscular e flexibilidade após aplicação da técnica. De forma semelhante, Irez et al. (2011) constataram melhora na flexibilidade e na força de membros inferiores em estudo realizado com 60 idosas saudáveis que praticaram Pilates 3 vezes por semana durante 12 semanas. Sinzato et al. (2013) apresentam em seu estudo que o método Pilates é capaz de melhorar a flexibilidade corporal geral, o alinhamento postural e a coordenação motora, além do aumento da força muscular, que está relacionado com uma melhora do controle motor e do recrutamento muscular.

No presente estudo, foi observada redução do tempo da caminhada de $2,44 \mathrm{~m}$ em todos os pacientes após a intervenção, corroborando os achados do estudo realizado por Johnson et al. (2013), no qual 10 pacientes com DP foram submetidos a 6 semanas de exercícios de Pilates, sendo observada melhora na mobilidade, no equilíbrio, na velocidade de caminhada e na cadência do passo desses pacientes.

RBCEH, Passo Fundo, v. 14, n. 2, p. 183-194, maio/ago. 2017 
Os exercícios do método Pilates selecionados neste estudo objetivaram: ganho de mobilidade de coluna vertebral, tronco e quadril; fortalecimento do power-house; melhora no ritmo escapuloumeral; e exercícios que desafiavam o equilíbrio e a coordenação, semelhante ao repertório de exercícios utilizado nos estudos de King e Horak (2009) e De Almeida e Ferraz (2009).

A amostra deste estudo foi composta por idosos que não praticavam atividade física por, no mínimo, dois meses, sendo utilizado o estímulo verbal durante os exercícios, bem como imagens para o entendimento da técnica e a facilitação do aprendizado motor. De Almeida e Ferraz (2009) também sinalizam a importância de estímulos cognitivos associados à atividade motora, para uma constante ativação da memória relacionada ao movimento em idosos parkinsonianos.

Estudos estrangeiros demonstram melhora dos marcadores funcionais, como velocidade da marcha, equilíbrio, qualidade de vida e padrão da marcha, em idosos com DP submetidos ao método Pilates (ROYER; WALDMANN, 2007; DE ALMEIDA; FERRAZ, 2009; KING; HORAK, 2009; BARTOLO et al., 2010; DE FREITAS; ZAGER; CAMPBELL, 2015). Em contrapartida, não foram encontrados artigos brasileiros que descrevessem os efeitos deste método na aptidão física dos idosos com DP. Dessa maneira, o presente estudo está entre as primeiras produções bibliográficas no Brasil que apresentam uma abordagem sobre o tema.
O presente estudo demonstra limitações devido à ausência de um grupo controle e ao número reduzido da amostra, o que não permitiu a realização de uma análise inferencial dos dados. Além disso, a literatura científica sobre aptidão física em idosos é escassa, o que limitou a discussão dos achados. Sugere-se a realização de ensaios clínicos randomizados com grupo de comparação, para observação de relação de causa e efeito do método Pilates na aptidão física de idosos com DP.

\section{Conclusão}

Os idosos com DP deste estudo apresentaram melhores parâmetros da aptidão física, quando submetidos à intervenção pelo método Pilates. Sugere-se, assim, que o método Pilates pode ser uma ferramenta eficaz no processo de reabilitação dos idosos parkinsonianos.

\section{The physical fitness of elderly people with Parkinson's disease in intervention by the Pilates method}

\section{Abstract}

Parkinson's disease (PD) is a neurodegenerative disease that promotes deterioration of the physical parameters of the elderly resulting in functional limitation and reduction of physical fitness. The Pilates methodis a modality of physical exercise that promotes benefits for the elderly, however, there is as hortage of scientific publication son the impact the technique on physical fitness parameters in elderly with PD. The objective of this study was to examine the 
physical fitness of elderly people with PD who under went Pilates intervention. This was a single-arm clinical trial performed with four elderly patients at the Physiotherapy Outpatient Clinic of a Geriatrics and Gerontology Center in Salvador, Bahia, Brazil. Elderly ( $\geq 60$ years) with a diagnosis of $\mathrm{PD}$, in regular use of the medications, and were not performing physical activity in the last two months prior to the intervention were included. Elderly patients with cognitive decline, musculoskeletaldys functions that compromised the performance of exercises, or irregular frequency ofintervention (three consecutive absences) were excluded. Data collected: clinical and sociodemographic, cognitive status, severity of PD and physical fitness. The elderly were submitted to the Pilates method (Pre-Pilates, Mat Pilates, balance and gait training, body relaxation exercises) for 30 sessions. After an intervention, values higher than the initial evaluation of upper and lower strength and force parameters, upper and lower limb flexibility, and physical mobility were observed. It is concluded that the Pilates method can be an effective tool in the rehabilitation process of elderly parkinsonians.

Keywords: Aged. Exercise movement techniques. Parkinson disease. Physical fitness.

\section{Referências}

ALENCAR, N. A. et al. Nível de atividade física, autonomia funcional e qualidade de vida em idosas ativas e sedentárias. Fisioterapia em Movimento, Curitiba, PR, v. 23, n. 3, p. 473-481, 2010.

BARTOLO, M. et al. Four-week trunkspecific rehabilitation treatment improves lateral trunk flexion in Parkinson's disease. Movement Disorders, Nova Iorque, v. 25, n. 3, p. 325-331, Feb. 2010.

BERTOLUCCI, P. H. et al. O mini-exame do estado mental em uma população geral: im- pacto da escolaridade. Arquivos de Neuropsiquiatria, São Paulo, SP, v. 52, p. 1-7, 1994.

CIPRIANI, N. C. S. et al. Aptidão funcional de idosas praticantes de atividades físicas. Revista Brasileira de Cineantropometria e Desempenho Humano, Florianópolis, SC, v. 12 , n. 2 , p. 106-111, 2010.

COSTA, L. M. R. et al. Os efeitos do método Pilates aplicado à população idosa: uma revisão integrativa. Revista Brasileira de Geriatria e Gerontologia, Rio de Janeiro, RJ, v. 19, n. 4, p. 695-702, 2016.

DA ROSA, K. B. et al. Qualidade de vida e avaliação funcional em idosos praticantes de Pilates e idosos sedentários. Revista Interdisciplinar de Estudos em Saúde, Florianópolis, SC, v. 2, n. 1, p. 18-28, 2013.

DE ALMEIDA, C. M. A.; FERRAZ, F. T. Uma abordagem da aplicação do Método Pilates na melhoria da qualidade de vida do idoso acometido da doença de Parkinson. In: CONGRESSO NACIONAL DE EXCELÊNCIAEM GESTÃO, 5, 2009, Niterói. Anais... Niterói: Firjan-RJ, 2009. p. 1-7.

DE FREITAS, M. L. M.; ZAGER, M.; CAMPBELL, C. A influência do método Pilates na instabilidade postural e qualidade de vida do paciente com doença de Parkinson. Fisioterapia Brasil, Rio de Janeiro, RJ, v. 16, n. 2, p. 155-159, 2015.

FREITAS, E. V. et al. Tratado de geriatria e gerontologia. Rio de Janeiro: Guanabara Koogan, 2006.

GALHARDO, M. M. A. M. C.; AMARAL, A. K. F. J.; VIEIRA, A. C. C. Caracterização dos distúrbios cognitivos na doença de Parkinson. Speech, Language, Hearing Sciences and Education Journal, São Paulo, SP, v. 11, n. 2, p. 251-257, 2009.

GONÇALVES, M. B. K.; ANGELO, R. C. O.; MARTINS, P. P. C. Aspectos clínicos e morfofuncionais da casa de força no método Pilates. Fisioterapia Brasil, Rio de Janeiro, RJ, v. 10, n. 1, p. 54-58, 2009.

RBCEH, Passo Fundo, v. 14, n. 2, p. 183-194, maio/ago. 2017 
GOULART, F. et al. Análise do desempenho funcional em pacientes portadores de doença de Parkinson. Acta Fisiátrica, São Paulo, SP, v. 11, n. 1 , p. 12-16, 2004.

GOULART, F.; PEREIRA, L. X. Uso de escalas para avaliação da doença de Parkinson em fisioterapia. Fisioterapia e Pesquisa, São Paulo, SP, v. 11, n. 1, p. 49-56, 2005.

GUIMARÃES, A. C. A. et al. Efeito do método Pilates na flexibilidade de idosos. Fisioterapia em Movimento, Curitiba, PR, v. 27, n. 2, p. 181-188, 2014.

IREZ, G. B. et al. Integrating Pilates exercise into an exercise program for $65+$ year-old women to reduce falls. Journal of Sports Science \& Medicine, Bursa, v. 10, n. 1, p. 105-111, Mar. 2011.

JOHNSON, L. et al. The effects of a supervised Pilates training program on balance in Parkinson's disease. Advances in Parkinson's Disease, v. 2, n. 2, p. 58-61, 2013.

KING, L. A.; HORAK, F. B. Delaying mobility disability in people with Parkinson disease using a sensorimotor agility exercise program. Physical Therapy, New York, v. 89, n. 4, p. 384-393, 2009.

LIMA, A. M. A. et al. Características clínicas e prevalência de sintomas depressivos em pacientes com a doença de Parkinson. Revista Brasileira de Ciências do Envelhecimento Humano, Passo Fundo, RS, v. 6, n. 2, p. 276283, maio/ago. 2010.

LIMA, M. do C. C. et al. Doença de Parkinson: alterações funcionais e potencial aplicação do método Pilates. Revista Brasileira de Geriatria e Gerontologia, Rio de Janeiro, RJ, v. 3, n. 1, p. 33-40, 2009.

LOBO, A.; PEREIRA, A. Idoso institucionalizado: funcionalidade e aptidão física. Revista Referência, Coimbra, v. 2, n. 4, p. 61-68, 2007.

LOURENÇO, R. A.; VERAS, R. P. Mini-Exame do Estado Mental: características psicométricas em idosos ambulatoriais. Revista de Saúde Pública, São Paulo, SP, v. 40, n. 4, p. 712-719, 2006.

MARÉS, G. et al. A importância da estabilização central no método Pilates: uma revisão sistemática. Fisioterapia em Movimento, Curitiba, PR, v. 25, n. 2, p. 445-451, 2012.

MELlO, M. P. B.; BOTELHO A. C. G. Correlação das escalas de avaliação utilizadas nadoença de Parkinson com aplicabilidade na fisioterapia. Fisioterapia em Movimento, Curitiba, PR, v. 23, n. 1, p. 121-127, 2010.

NASCIMENTO, M. de M.; LIMA, R. K. R. Envelhecendo equilibradamente: considerações de um programa de atividade física para idosos fundamentado no método Pilates. Extramuros, Petrolina, v. 1, n. 1, p. 108-123, 2013.

NOGUEIRA, S. L. et al. Fatores determinantes da capacidade funcional em idosos longevos. Revista Brasileira de Fisioterapia, São Carlos, SP, v. 14, n. 4, p. 322-329, 2010.

PEREIRA, F. D. et al. Comparação da força funcional de membros inferiores e superiores entre idosas fisicamente ativas e sedentárias. Revista Brasileira de Geriatria e Gerontologia, Rio de Janeiro, RJ, v. 12, n. 3, p. 417427, 2009.

PINHEIRO, I. de M. et al. Impacto da doença de Parkinson na funcionalidade e qualidade de vida de idosos em uma unidade de referência geriátrica na cidade de Salvador-Bahia. Revista de Ciências Médicas e Biológicas, v. 13, n. 3, p. 292-297, 2014.

PINHEIRO, K. R. G. et al. Influence of Pilates exercises on soil stabilization in lumbar muscles in older adults. Revista Brasileira de Cineantropometria \& Desempenho Humano, São Paulo, SP, v. 16, n. 6, p. 648-657, 2014.

QUINTELLA, R. S. et al. Qualidade de vida e funcionalidade na doença de Parkinson. Revista Brasileira de Ciências do Envelhecimento Humano, Passo Fundo, RS, v. 10, n. 1, p. 104-112, jan./abr. 2013. 
REIS, L. A.; MASCARENHAS, C. H. M.; LYRA, J. E. Avaliação da qualidade de vida em idosos praticantes e não praticantes do método Pilates. C\&D-Revista Eletrônica de Fainor, Vitória da Conquista, ES, v. 4, n. 1, p. 38-51, 2011.

RIKLI, R. E.; JONES, C. J. Fitness of older adults. The Journal on Active Aging, v. 2, n. 1, p. 24-30, Mar./Apr. 2002.

ROYER, L.; WALDMANN, K. Rehab Corner: Pilates for people with Parkinson's disease. Pilates Coreterly, v. 1, n. 1, p. 60-65, 2007.

SANT, C. A. et al. Abordagem fisioterapêutica na doença de Parkinson. Revista Brasileira de Ciências do Envelhecimento Humano, Passo Fundo, RS, v. 5, n. 1, p. 80-89, jan./ jun. 2008.

SEEMANN, T. et al. Treinabilidade e reversibilidade na aptidão física de idosas participantes de programa de intervenção. Revista Brasileira de Geriatria e Gerontologia, Rio de Janeiro, RJ, v. 19, n. 1, p. 129-137, 2016.

SINZATO, C. R. et al. Efeitos de 20 sessões do método Pilates no alinhamento postural e flexibilidade de mulheres jovens: estudo piloto. Fisioterapia e Pesquisa, São Paulo, SP, v. 20, n. 2, p. 143-150, 2013.

VILA, C. P. et al. Aptidão física funcional e nível de atenção em idosas praticantes de exercício físico. Revista Brasileira de Geriatria e Gerontologia, Rio de Janeiro, RJ, v. 16, n. 2, p. 355-364, 2013.

WERNECK, A. L. S.; ALVARENGA, H. Genetics, drugs and environmental factors in Parkinson's disease: a case-control study. Arquivos de Neuropsquiatria, São Paulo, SP, v. 57, n. 2, p. 347-355, 1999. 